INVESTIGACIÓN

\title{
CONTROL PROPORCIONAL INTEGRAL GENERALIZADO (GPI) PARA EL LAZO DE CORRIENTE DE UN CONVERTIDOR AC-DC BOOST BRIDGELESS
}

\section{GENERALIZED PROPORTIONAL INTEGRAL (GPI) CONTROL FOR A BOOST BRIDGELESS AC-DC CONVERTER CURRENT LOOP}

\author{
MSc.Fernando Jesús Regino Ubarnes ${ }^{\mathrm{a}}$, Ing. Edgar Modesto Ochoa ${ }^{\mathrm{b}}$, Ing. Andrea Lorena Vergel \\ Romero ${ }^{c}$ \\ ${ }^{a}$ Grupo de investigación GITYD, Universidad Francisco de Paula Santander Ocaña, Calle \\ 6b\#20-30, Ocaña, Colombia, fjreginou@ufpso.edu.co \\ ${ }^{\mathrm{b}}$ Grupo de investigación GITYD, Universidad Francisco de Paula Santander Ocaña, Casa 2 \\ Villas de Anton, Ocaña, Colombia, emochoaf@ufpso.edu.co \\ ${ }^{c}$ Grupo de investigación GIGMA, Universidad Francisco de Paula Santander Ocaña, Carrera 38 \\ \# 7a - 25, Ocaña, Colombia, alvergelr@ufpso.edu.co
}

Fecha de recepción: 11-03-2017

Fecha de aprobación: 15-06-2017

\begin{abstract}
Resumen: Este documento describe el modelamiento de un convertidor AC-DC y el diseño de una técnica de Control Proporcional Integral Generalizado (GPI). El diseño de dicha técnica se da en el contexto del rechazo activo de perturbaciones (ADRC), en el cual se muestra que las técnicas de control GPI son capaces de rechazar las perturbaciones de carácter endógeno y exógeno de la planta, lo que lleva a un error de seguimiento acotado y dicha cota puede hacerse tan pequeña como se quiera al alejar las raíces del eje imaginario.
\end{abstract}

Palabras clave: control proporcional integral generalizado, factor de potencia, distorsión armónica total.

\begin{abstract}
This paper describes the modeling of an AC-DC converter and the design of a Generalized Integral Proportional Control (GPI) technique. The design of this technique is given in the context of Active Disruption Disorder (ADRC), which shows that GPI control techniques are able to reject endogenous and exogenous perturbations of the plant, leading to a bounded error which can be as small as desired the by getting apart the roots from the imaginary axis.
\end{abstract}

Keywords: Generalized integral proportional control, power factor, total harmonic distortion 


\section{INTRODUCCIÓN}

Los convertidores de potencia AC-DC con corrección de factor de potencia (PFC) son muy comunes en aplicaciones que requieran trabajar con una tención de corriente directa, su aplicación en los sistemas de alimentación ininterrumpida (UPS), servidores de tipo industrial, drivers de motores DC, entre otros, es significativa. En este tipo de sistemas el factor de potencia (FP) y la distorsión armónica total (THD) son los criterios que se tienen en cuenta para evaluar la calidad de la energía(Daniali, 2017).

Hoy en día, las corrientes armónicas están aumentando debido al crecimiento de las cargas no lineales en los sistemas de distribución. Corrientes armónicas Conducir a la reducción de la calidad de la energía Dispositivos, aumentando las pérdidas en los transformadores, y las máquinas eléctricas, Creación de estrés en los bancos de condensadores, mal funcionamiento de dispositivos de protección, etc. La localización y la determinación de nivel de las fuentes armónicas son los primeros pasos para resolver estos problemas. La literatura sobre la detección de fuentes armónicas puede clasificarse en dos grupos: métodos de un solo punto y métodos de múltiples puntos. Los métodos de punto único determinan sólo la fuente armónica dominante en el punto de medición.

Las cargas no lineales crean corrientes armónicas que se propagan en las redes de transmisión y distribución eléctrica, las corrientes armónicas reducen la calidad de la energía reduciendo la vida útil de los dispositivos. Las corrientes armónicas aumentan las pérdidas en los transformadores y en las máquinas eléctricas, crean estrés en los bancos de condensadores y mal funcionamiento de dispositivos de protección; Además causan sobrecalentamiento en las líneas y transformadores de distribución (Emannuel, Horton, Jewel, \& Phileggi, 1993)(Moradifar, Foroud, \& Firouzjah, 2017).

Los estándares IEC1000-3-2 y EN61000-3-2 regulan los armónicos en la línea de alimentación (Lazaro, Barrado, Sanz, Salas, \& Olias, 2007), el estándar IEEE519 que regulan los armónicos en la red eléctrica, limita el porcentaje del THD de la señal de corriente de entrada a un máximo del 5\% (Patel, Pixler, \& Nasiri, 2010). Los convertidores AC-DC conmutados adoptan controladores para hacer corrección de factor de potencia (PFC) esto para aprovechar toda la potencia activa de la carga llevando el FP a un valor muy cercano a la unidad.

Después de la sección 1 que ofrece una introducción, la sección 2 presenta el modelo matemático de un convertidor $\mathrm{AC}$ DC Boost Bridgeless. En la sección 3 se presenta una descripción de la estrategia de control GPI. La sección cuatro muestra el diseño de dicha estrategia para el modelo promediado simplificado del convertidor AC-DC Boost Bridgeless. La sección cinco muestra los resultados de la simulación del arreglo propuesto y la sección seis cubre una conclusión.

\section{METODOLOGÍA}

La dinámica del convertidor está regida por el tiempo de encendido y apagado los Mosfet Q1 y Q2, estos establecen el recorrido de la corriente. En el semi-ciclo positivo la señal de PWM actúa directamente sobre Q1 mientras Q2 
permanece apagado, en el semi-ciclo negativo la señal de PWM actúa directamente sobre Q2 mientras Q1 permanece apagado.

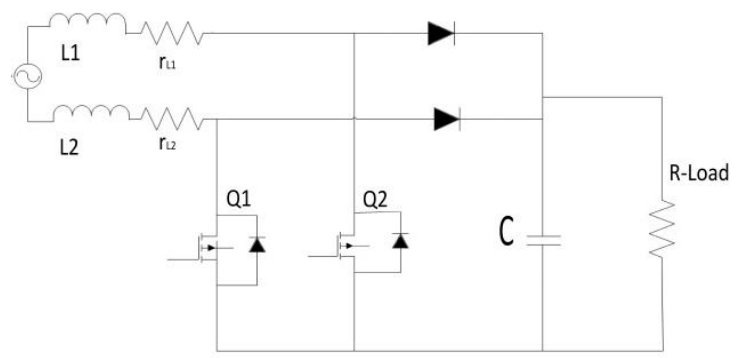

Figura 1 Diagrama esquemático del PFC Boost Bridgeless

La figura 13 muestra recorrido de la corriente en el semi-ciclo positivo con Q1 y Q2 apagados, es en este instante en donde se carga el capacitor $\mathrm{C}$; la figura 14 muestra recorrido de la corriente en el semi-ciclo positivo con Q1 encendido y Q2 apagado cargando las bobinas L1 y L2, La figura 15 muestra recorrido de la corriente en el semiciclo negativo con Q1 y Q2 apagados, cargando el capacitor $\mathrm{C}$; la figura 16 muestra recorrido de la corriente en el semiciclo negativo con Q1 apagado y Q2 encendido cargando las bobinas L1 y L2.

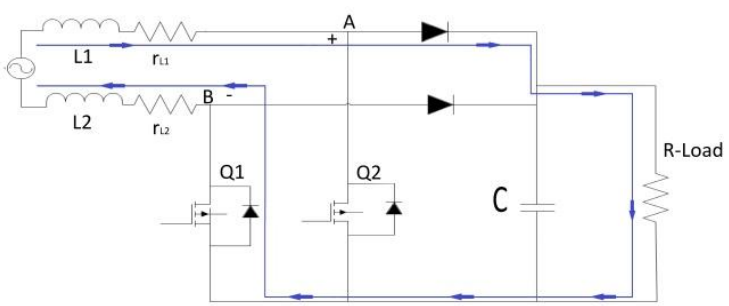

Figura 2 recorrido de la corriente en el semi-ciclo positivo con Q1 y $Q 2$ apagados

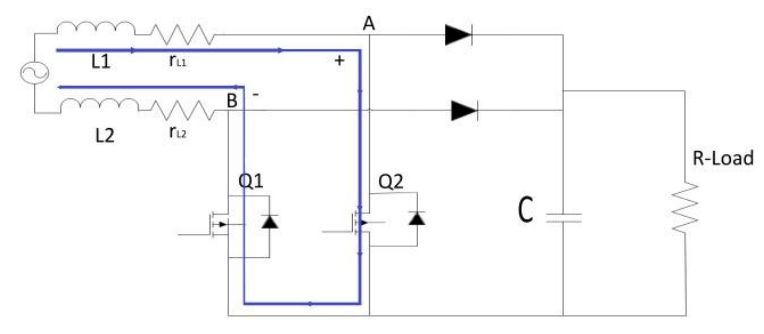

Figura 3 recorrido de la corriente en el semi-ciclo positivo con $Q 1$ encendido y $Q 2$ apagado

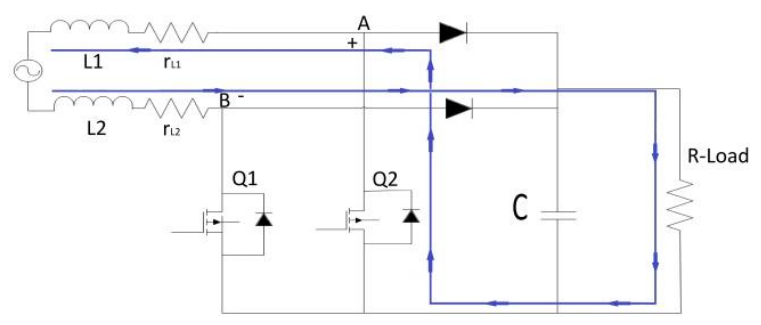

Figura 4 recorrido de la corriente en el semi-ciclo negativo con $Q 1$ y $Q 2$ apagados

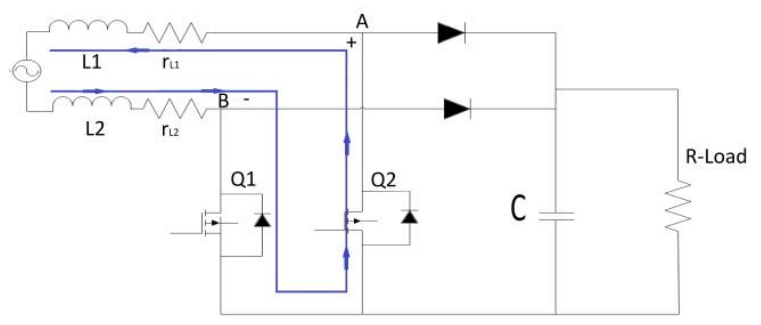

Figura 5 recorrido de la corriente en el semi-ciclo negativo con $Q 1$ apagado y $Q 2$ encendido

Se hace el análisis del circuito (ver figura 2) empleando la ley de tensiones de Kirchhoff.

$v_{f(t)}=L \frac{d i_{L(t)}}{d t}+R_{L} i_{L(t)}+v_{a b(t)}$

Donde $v_{f(t)}$ es la tensión de la red $\left(v_{f(t)}=\right.$ Vf2sinwnt, iLt es la corriente que pasa por la bobina, $L$ es la inductancia equivalente del circuito, $R_{L}$ es la resistencia equivalente del circuito igual $\mathrm{r}_{\mathrm{L} 1}+\mathrm{r}_{\mathrm{L} 2}$ y $v_{a b(t)}$ es la tensión vista desde los nodos A y B del circuito.

Cuando $v_{f(t)}$ es positiva la tensión $v_{a b(t)}$ promedio es:

$$
\begin{gathered}
\overline{v_{a b(t)}}=(1-D)\left(v_{c(t)}+v_{D 1}\right)+v_{Q 1} D \\
+v_{D Q 2}(2)
\end{gathered}
$$

Cuando $v_{f(t)}$ es negativa la tensión $v_{a b(t)}$ promedio es: 


$$
\begin{gathered}
\overline{v_{a b(t)}}=-(1-D)\left(v_{c(t)}+v_{D 2}\right)-v_{Q 2} D \\
-v_{D Q}(3)
\end{gathered}
$$

En donde $v_{D 1(t)}$ es el voltaje del diodo D1; $v_{c(t)}$ es el voltaje DC del capacitor de salida; $v_{D Q 2(t)}$ es el voltaje del diodo interno del Mosfet Q2; $v_{D Q 1(t)}$ es el voltaje del diodo interno del Mosfet Q1; $v_{Q 1(t)}$ es la tensión drenador-surtidor del Mosfet Q1; $v_{Q 2(t)}$ es la tensión drenador-surtidor del Mosfet Q2.

En las ecuaciones 2 y 3, D corresponde al ciclo útil de la señal de PWM que toma valores en un intervalo cerrado de $[0,1]$.

Al sumar las ecuaciones 2 y 3 es posible afirmar que el modelo promediadosimplificado de la planta es:

$\frac{d i_{L(t)}}{d t}=-\frac{R_{L}}{L} i_{L(t)}+\frac{v_{f(t)}}{L}+\frac{\alpha}{L}(4)$

Donde $\alpha$ es:

$=\left\{\begin{array}{c}(1-D) v_{c(t)} ; \text { semi }- \text { ciclo positivo } \\ -(1-D) v_{c(t)} ; \text { semi }- \text { ciclo negativo }\end{array}\right.$

Al aplicar la transformada de Laplace a la ecuación 4 , y tomando $v_{f}$ como una perturbación se obtiene la ecuación 6 .

$S I_{L}=-\frac{R_{L}}{L} I_{L}+\frac{\alpha}{L}(6)$

De la ecuación 6 se despeja $\frac{I_{L}}{\alpha}$ y da como resultado el modelo promediadosimplificado a la frecuencia de conmutación de la planta (Ecuación 7).

$\frac{I_{L}}{\alpha}=\frac{1}{\left(L s+R_{L}\right)}(7)$
Para la simulación de la planta se establecen valores para $L=707 \mu \mathrm{H}$ y para $R_{L}=1 \Omega$.

\section{Control proporcional integral generalizado (GPI)}

Los controladores GPI utilizan el modelo simplificado del sistema y rechazan las dinámicas no modeladas, perturbaciones de carácter endógeno y exógeno del sistema. El control GPI se diseña en el marco del rechazo activo de perturbaciones, en el cual los controladores GPI incluyen un modelo polinomial en el tiempo de las perturbaciones dependientes del estado y de aquellas perturbaciones de carácter exógeno sin estructura especial alguna (Cortésromero, 2015).

Esta técnica de control nos permite ver el problema de control como el control de una cadena de integradores, en donde el orden del polinomio y de la cadena integradores determina el número de parámetros de la ganancia integral de una manera simple (Luviano-Juarez, Cortes-Romero, \& SiraRamirez, 2008).

El objetivo de control es lograr la eliminación de las perturbaciones aditivas que afectan el sistema (Erickson \& W., n.d.). Se consideran sistemas lineales perturbados cuando su modelo es de la forma de la (ecuación 13).

$$
\begin{gathered}
y^{(n)}(t)=\kappa u(t)+\phi\left(t, y, \dot{y}, \ldots, y^{(n-1)}\right) \\
+\varsigma(t)(13)
\end{gathered}
$$

Donde $\boldsymbol{\phi}$ agrupa la incertidumbre del sistema es decir las dinámicas lineales no modeladas o desconocidas y $\boldsymbol{\varsigma}$ representa la perturbación externa (Fliess, Lévine, Martin, \& Rouchon, 1995). Desde el enfoque del rechazo activo de perturbaciones, estas dos funciones se consolidan en un solo término y se maneja como una señal de carácter desconocido pero acotado (ecuación 14). 
$\boldsymbol{y}^{(\boldsymbol{n})}(\boldsymbol{t})=\boldsymbol{\kappa u}(\boldsymbol{t})+\xi(t)$

Donde $\quad \xi(\boldsymbol{t})$ es m-diferenciable y uniformemente acotada, i.e. $\sup \left|\xi^{(\boldsymbol{m})}(\boldsymbol{t})\right| \leq$ $\boldsymbol{K}$, con $\mathrm{K}$ real finito. El sistema es controlable (para $\kappa \neq 0$ ) y también puede ser catalogado como un sistema diferencialmente plano con salida plana $\boldsymbol{y}(\boldsymbol{t})$ (Sira-Ramírez \& Agrawal, 2004).

Como se puede ver en la ecuación 7 el modelo de la planta se puede expresar en función de las derivadas de la salida del sistema, por lo que se puede tomar la salía del sistema como una salida plana.

Teniendo en cuenta que el modelo matemático del convertidor es el que se muestra en la siguiente ecuación.

$\frac{d i_{L(t)}}{d t}=-\frac{R_{L}}{L} i_{L(t)}+\frac{v_{f(t)}}{L}+\frac{\alpha}{L}$

Es posible afirmar que dicho modelo tiene la forma del sistema descrito por la ecuación 14.

Donde $\xi(t)=-\frac{R_{L}}{L} i_{L(t)}+\frac{v_{f(t)}}{L}$ у $\kappa u(t)=$ $\frac{\alpha}{L}$

En la ecuación 16 se muestra la estructura del control GPI, en donde $\kappa$ es $1 / \mathrm{L}$ y la salida $Y$ del sistema es la corriente $i_{L}$ que atraviesa las bobinas L1 y L2; $m$ es el orden del polinomio con el que se aproxima la perturbación; $n$ es el orden del sistema; $\mathrm{K}_{\mathrm{n}+\mathrm{m}}, \ldots, \mathrm{K}_{1}, \mathrm{~K}_{0}$ corresponden las ganancias del polinomio del numerador; y $Y^{*}$ es la señal de referencia que para este caso equivale a una senoidal con frecuencia de $60 \mathrm{~Hz}$ y amplitud 10 amperios pico.

\section{Diseño del controlador}

El control GPI se diseña a partir de la estructura de la señal de control (ecuación 15). Para este diseño se parte del supuesto que la $m$-ésima derivada de la perturbación con respecto al tiempo es cero y se toma $m=1, n=1$ la ecuación 15 se puede escribir como:

$U=$

$\frac{1}{\kappa}\left[y^{*(n)}+\right.$

$K n+m s n+m+\ldots+K 1 s+K 0 s m+1 s n-1+K$ $2 n+m-1 s n-2+\ldots K n+m+1 y *-y(15)$

Para el caso de estudio la estructura del control GPI se puede escribir como:

$U=\frac{1}{\mathrm{~K}}\left[y^{*(1)}+\left(\frac{K_{3} s^{3}+K_{2} s^{2}+K_{1} s+K_{0}}{s^{3}}\right)\left(y^{*}-\right.\right.$ $y(16)$

En la figura 6 se muestra el diagrama de bloques del sistema que se obtiene en lazo cerrado al aplicar el control GPI que se describe en la ecuación 16.

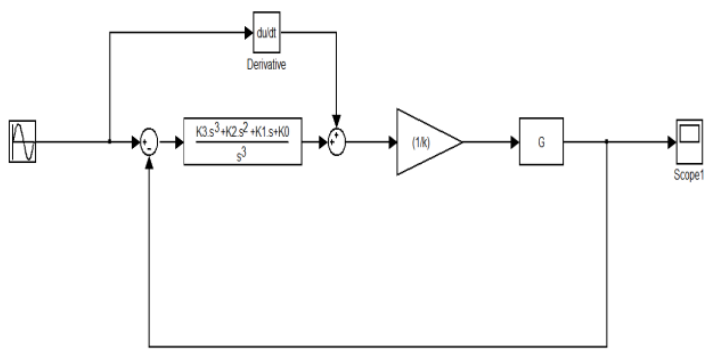

Figura 6 Diagrama de bloques del control GPI para $m 2$.

El control GPI se diseña a partir de la señal de control (ecuación 16). Para este diseño se parte del supuesto que la $m$-ésima derivada de la perturbación con respecto al tiempo es cero y se toma $m=2, n=1$ la ecuación 14 se puede escribir como:

$\dot{\mathrm{y}}=\mathrm{ku}+\xi$

Reemplazando la ecuación 16 en la ecuación 17, se aplica la transformada de Laplace y se despeja $\xi$ resultando en la ecuación 18 
$s\left(Y-Y^{*}\right)+\left(\frac{K_{3} s^{3}+K_{2} s^{2}+K_{1} s+K_{0}}{s^{3}}\right)(Y-$

$Y *=\xi(18)$

Donde $\left(Y-Y^{*}\right)$ es el error de seguimiento y $s\left(Y-Y^{*}\right)$ es la derivada del error de seguimiento: $E=\left(Y-Y^{*}\right) \quad$ y $\quad s E=$ $s\left(Y-Y^{*}\right)$

Reemplazando el error $E$ y la derivada del error $(s E)$ en la ecuación 18 , resulta en la ecuación 19.

$s E+\left(\frac{K_{3} s^{3}+K_{2} s^{2}+K_{1} s+K_{0}}{s^{3}}\right) E=\xi$

De la ecuación 19 se despeja $s^{4} \xi$ y resulta la ecuación 20.

$\left(s^{4}+K_{3} s^{3}+K_{2} s^{2}+K_{1} s+K_{0}\right) E=s^{4} \xi$

La ecuación 20 muestra la dinámica del error, si $\xi(t)^{(4)}$ es acotada y si los coeficientes $K_{3}, K_{2}, K_{1}$ y $K_{0}$ se escogen de tal forma que las raíces del polinomio de la ecuación 20 estén en el lado izquierdo del plano complejo, el error de seguimiento será acotado y la cota puede hacerse tan pequeña como se quiera al alejar las raíces de la ecuación 21 del eje imaginario.

$s^{4}+K_{3} s^{3}+K_{2} s^{2}+K_{1} s+K_{0}(21)$

Para encontrar los valores de $K_{3}, K_{2}, K_{1}$ y $K_{0}$ se ubican los polos en lazo cerrado en [-20 $30-40$-50], de esta forma se garantiza la estabilidad del sistema $\mathrm{y}$ un buen desempeño.

\section{RESULTADOS}

En la figura 7, se muestran las gráficas de corriente y referencia de corriente obtenidas en la simulación de la planta sin de control, a la planta se le aplica como perturbación una señal tipo paso a los 0.23 segundos, se evidencia que los resultados en cuanto al seguimiento de la señal de referencia no rechaza este tipo de perturbaciones.

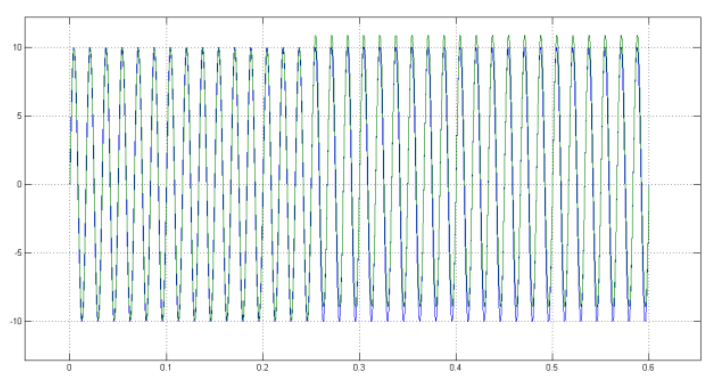

Figura 7 gráficas de corriente y referencia de corriente.

En la figura 8, se muestran las gráficas de corriente y referencia de corriente obtenidas en la simulación de la planta con control GPI, a la planta se le aplica como perturbación una señal tipo paso a los 0.23 segundos, se evidencia que los resultados en cuanto al seguimiento de la señal de referencia que el controlador GPI con polos en $\left[\begin{array}{llll}-20 & -30 & -40 & -50\end{array}\right]$ rechazan este tipo de perturbaciones, aunque presenta un sobrepico transitorio de aproximadamente el 40.

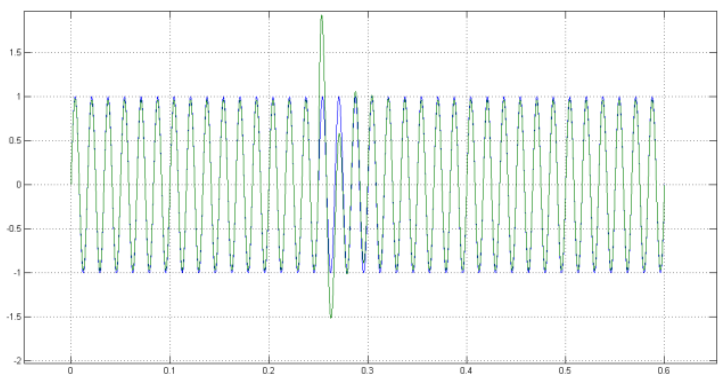

Figura 8 gráficas de corriente y referencia de corriente con control GPI.

En la figura 9, se muestran las gráficas de corriente y referencia de corriente obtenidas en la simulación de la planta con control GPI, a la planta se le aplica como 
perturbación una señal tipo paso a los 0.23 segundos, se evidencia que los resultados en cuanto al seguimiento de la señal de referencia que el controlador GPI con polos en [-200 -300 -400 -500] rechazan este tipo de perturbaciones, aunque presenta un sobrepico transitorio de aproximadamente el $1 \%$.

\section{CONCLUSIONES}

Se puede decir que las estrategias de control GPI provén una herramienta efectiva para el rechazo activo de perturbaciones, las cuales dependen de la ubicación de los polos, entre más alejados estén del eje imaginario mejor será el seguimiento de la señal de referencia y el rechazo de perturbaciones.

\section{BIBLIOGRAFÍA}

Cortés-romero, J. A. (2015). Control GPIrepetitivo para sistemas lineales con incertidumbre / variación en los parámetros GPI-repetitive control for linear systems with parameter uncertainty / variation, 18(34), 13-24.

Daniali, N. (2017). High Efficiency LLCBased AC-DC Converter for Wide Load Voltage Range Applications, (May), 16-18.

Emannuel, a E., Horton, W. F., Jewel, W. T., \& Phileggi, D. J. (1993). Effects of Harmonics on Equipment Report of the IEEE Task Force on the Effects of Harmonics on Equipment. IEEE Transactions on Power Delivery, 8(2), 672-680.

https://doi.org/10.1109/61.216874

Erickson, Y. L., \& W., H. K. R. (n.d.). Design of EMI Filters Having Low Harmonic Distortion in High-PowerFactor Converters. Retrieved November 21, 2015, from http://ieeexplore.ieee.org.ezproxy.unal. edu.co/xpls/icp.jsp?arnumber $=6594916$

Fliess, M., Lévine, J., Martin, P., \& Rouchon, P. (1995). Flatness and defect of non-linear systems: introductory theory and examples. International Journal of Control, 61(6), 1327-1361. https://doi.org/10.1080/0020717950892 1959

Lazaro, A., Barrado, A., Sanz, M., Salas, V., $\&$ Olias, E. (2007). New Power Factor Correction AC-DC Converter With Reduced Storage Capacitor Voltage. IEEE Transactions on Industrial Electronics, 54(1), 384-397. https://doi.org/10.1109/TIE.2006.88879 5

Luviano-Juarez, A., Cortes-Romero, J., \& Sira-Ramirez, H. (2008). Chaotic synchronization between oscillators using robust GPI control. 2008 th International Conference on Electrical Engineering, Computing Science and Automatic Control, (Cce), 114-119. https://doi.org/10.1109/ICEEE.2008.47 23451

Moradifar, A., Foroud, A. A., \& Firouzjah, K. G. (2017). Intelligent localisation of multiple non-linear loads considering impact of harmonic state estimation accuracy. IET Generation, Transmission and Distribution, 11(8), 1943-1953. https://doi.org/10.1049/ietgtd.2016.1278

Patel, Y., Pixler, D., \& Nasiri, A. (2010). Analysis and design of TRAP and LCL filters for active switching Converters. IEEE International Symposium on Industrial Electronics, 638-643. https://doi.org/10.1109/ISIE.2010.5637 475

Sira-Ramírez, H., \& Agrawal, S. K. (2004). 
Differentially Flat Systems. Retrieved from

https://books.google.com/books?hl=es $\& \mathrm{lr}=\& \mathrm{id}=\mathrm{IlknugDBYY}$ 8C \& pgis $=1$ 